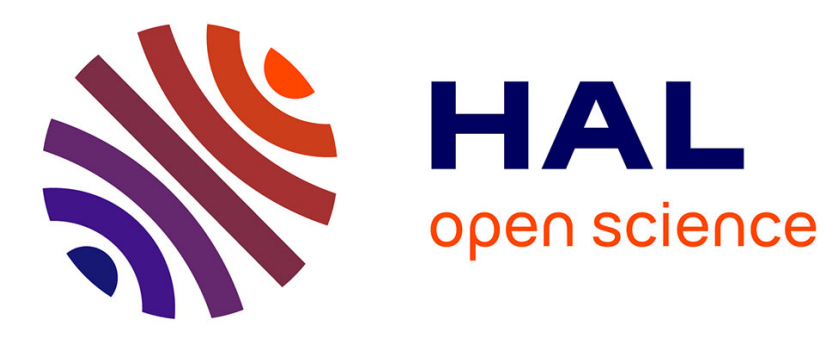

\title{
Renforcer les capacités réflexives à des fins de développement professionnel: une grille-repères pour la conception de dispositifs pédagogiques
}

Lucie Gouttenoire, Cécile Fiorelli, Laurent Trognon, Christèle Roux

\section{> To cite this version:}

Lucie Gouttenoire, Cécile Fiorelli, Laurent Trognon, Christèle Roux. Renforcer les capacités réflexives à des fins de développement professionnel: une grille-repères pour la conception de dispositifs pédagogiques. Revue Phronesis, 2019, Phronesis, 8 (3-4), pp.48-61. hal-02457379

\section{HAL Id: hal-02457379 \\ https://hal.science/hal-02457379}

Submitted on 3 Mar 2020

HAL is a multi-disciplinary open access archive for the deposit and dissemination of scientific research documents, whether they are published or not. The documents may come from teaching and research institutions in France or abroad, or from public or private research centers.
L'archive ouverte pluridisciplinaire HAL, est destinée au dépôt et à la diffusion de documents scientifiques de niveau recherche, publiés ou non, émanant des établissements d'enseignement et de recherche français ou étrangers, des laboratoires publics ou privés. 


\title{
Renforcer les capacités réflexives à des fins de développement professionnel : une grille-repères pour la conception de dispositifs pédagogiques
}

\author{
Lucie GOUTTENOIRE*, Cécile FIORELLI*, Laurent TROGNON* et Christèle ROUX**
}

\begin{abstract}
*Université Clermont-Auvergne, AgroParisTech, Inra, Irstea, VetAgro Sup, UMR Territoires, Clermont-Ferrand, France lucie.gouttenoire@vetagro-sup.fr; cecile.fiorelli@inra.fr; laurent.trognon@agroparistech.fr

${ }^{\star \star}$ Agrosup Dijon, Eduter, France

christele.roux@agrosupdijon.fr
\end{abstract}

Mots-clés : réflexivité, dispositifs pédagogiques, développement, situations professionnelles, étayage

Résumé : Alors que le concept de réflexivité se présente comme incontournable en formation professionnelle, les repères pour construire des dispositifs pédagogiques visant le renforcement des capacités réflexives d'acteurs sur leurs situations professionnelles à des fins de développement professionnel et personnel paraissent, sinon manquants, du moins noyés dans un foisonnement théorique et pratique. En tant que concepteurs de tels dispositifs dans le domaine de l'enseignement supérieur agronomique, nous présentons une grille-repères articulant cinq dimensions de la réflexivité, forgée au fil de nos expériences, de nos échanges et de nos lectures. Ces cinq dimensions renvoient à cinq aspects de la réflexivité qu'il est possible de travailler directement avec des apprenants, en mettant en place un étayage adapté que nous caractérisons et illustrons dans l'article, en puisant dans nos expériences de formateurs. Deux dimensions de la grille sont particulièrement pertinentes pour accompagner des publics non familiers avec la réflexivité, et parfois réfractaires aux exercices réflexifs.

\section{Title : Enhancing reflective capacities to foster professional development: a grid to design pedagogical devices}

Keywords: reflexivity, pedagogical devices, development, professional situations, scaffolding

\begin{abstract}
While reflexivity is seen as a not-to-be-missed concept in professional training, references to design pedagogical devices to enhance actors' reflective capacities on their professional situations and to foster their professional and personal development are scarce and embedded in a patchwork of theoretical and practical approaches. As designers of such devices in agricultural higher education, our practical experiences, theoretical background and discussions led us to build a model of reflexivity as five intertwined key dimensions. These dimensions deal with aspects of reflexivity that it is possible to work on directly with learners by using appropriate scaffolding methods, which we describe and illustrate in this paper, referring to our teaching experiences. Two dimensions of the model are particularly relevant for people who are not familiar with reflexivity, and sometimes reluctant to practice it.
\end{abstract}


Les enseignants ne perçoivent pas toujours l'enjeu de construire des dispositifs permettant de renforcer les capacités réflexives des apprenants : beaucoup pensent qu'il suffit de fréquenter des situations de travail pour acquérir les compétences professionnelles, idée déconstruite, par exemple, par Donnadieu (2011). Et quand ils perçoivent cet enjeu, quelles ressources sont à leur disposition pour construire, questionner et améliorer leurs dispositifs? Rares sont les travaux, qui comme ceux de Faingold (2015), donnent à voir un dispositif dans sa globalité, les objectifs et les hypothèses sous-jacentes du concepteur de dispositif, ainsi que la manière d'articuler différents moments, et sont ainsi susceptibles de nourrir de telles réflexions. La littérature est riche en récits de pratique professionnelle et en résultats de recherche sur l'usage de différents outils (Caron et Chouinard, 2015), par exemple les écrits réflexifs (Morisse, 2014), le récit professionnel (Beaujouan, Coutarel, Daniellou, 2013) ou encore les groupes d'analyse de pratiques professionnelles (Fablet, 2004). Ces travaux permettent certes d'enrichir la boîte à outils des concepteurs de dispositifs, mais nous sont apparus insuffisants pour questionner la cohérence et la pertinence de dispositifs visant le développement des capacités réflexives d'apprenants sur leurs situations professionnelles, à des fins de développement. En particulier, notre réflexion porte sur les dispositifs à mettre en œuvre pour accompagner des publics hétérogènes, pour partie non familiers, voire réfractaires à l'activité réflexive.

Faingold (2015) explique que c'est en écrivant son article qu'elle a fait émerger la logique sous-jacente et la progression de son dispositif. De notre côté, soucieux de prendre du recul sur nos dispositifs, de les améliorer et de contribuer aux réflexions sur la conception de dispositifs de renforcement des capacités réflexives à des fins de développement, nous présentons dans cet article une grille-repères articulant cinq dimensions de la réflexivité, forgée au fil de nos expériences, de nos échanges et de nos lectures. Dans un premier temps, nous rendons compte de la démarche suivie pour formaliser cette grille à partir de l'analyse croisée de trois dispositifs d'accompagnement que nous portons depuis plusieurs années. Nous présentons ensuite chacune des cinq dimensions. Enfin nous discutons en quoi cette grille-repères contribue à donner davantage de corps et de consistance à la notion de réflexivité en formation.

\section{Construction de la grille-repères}

La méthodologie qui sous-tend cette recherche-action se compose de deux étapes. Durant la première, trois dispositifs pédagogiques distincts ont été menés de manière indépendante par les auteurs de cet article. À la suite d'une série d'échanges permis par la création nouvelle d'un collectif de recherche, la pertinence d'élaborer un outil synthétisant nos travaux empiriques a été examinée, suite à quoi nous avons enclenché l'étape 2 qui a consisté en une analyse croisée de nos trois dispositifs, puis la construction et la validation de la grille-repères présentée plus loin. L'ensemble de la démarche suivie est représenté en figure 1 et détaillé ci-après.

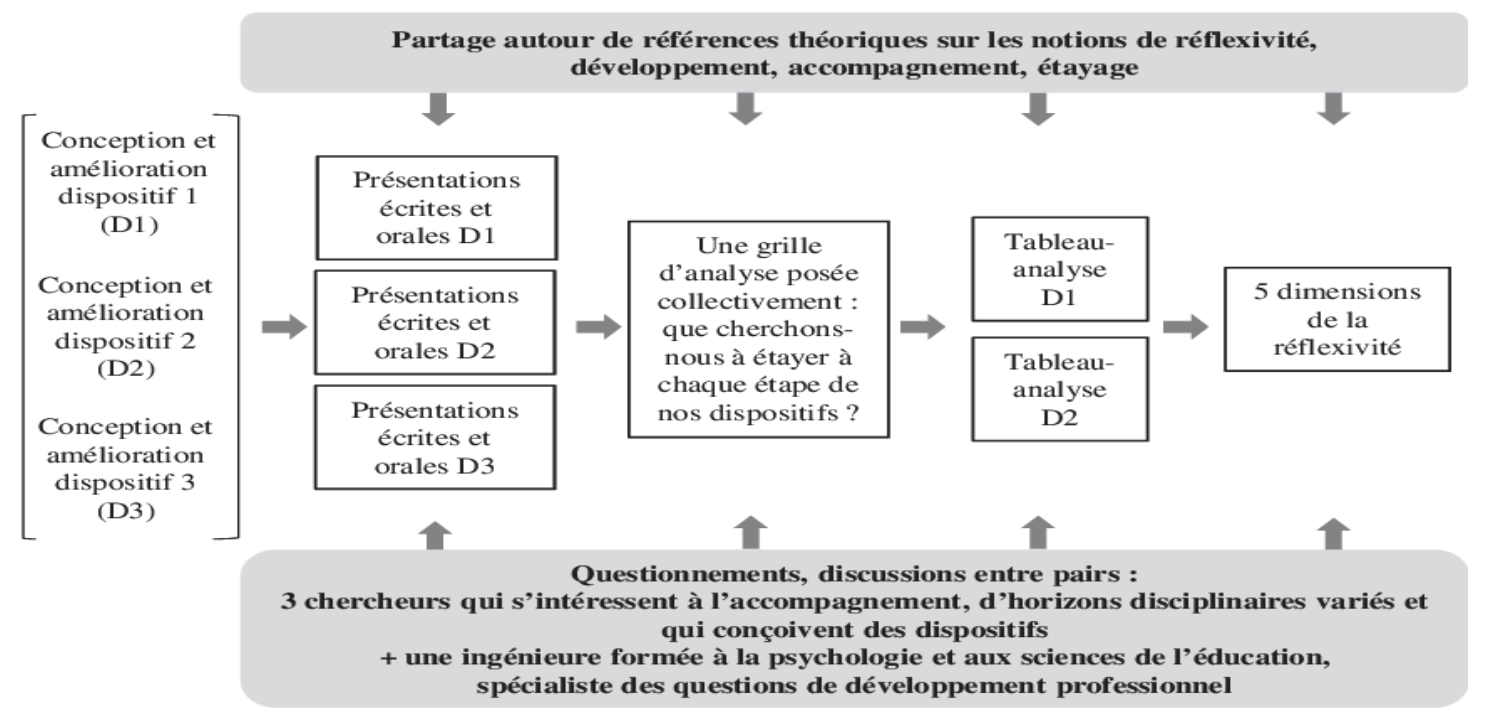

Figure 1. Démarche méthodologique. 


\section{Trois dispositifs pédagogiques support d"une analyse croisée}

Les trois dispositifs concernent respectivement des ingénieurs agronomes en formation initiale (dispositif 1, nommé D1 par la suite), des doctorants du département Sciences pour l'Action et le Développement de I'Institut National de la Recherche Agronomique (INRA) (dispositif 2, nommé D2 par la suite), et des agents de développement territorial en formation continue (dispositif 3 nommé D3 par la suite). Chacun des trois premiers auteurs de cet article porte ou co-porte un de ces trois dispositifs, présentés dans le tableau 1.

\begin{tabular}{|c|c|c|c|c|c|c|c|c|}
\hline $\begin{array}{l}\frac{1}{4} \\
\frac{1}{n} \\
0 \\
0 \\
0 \\
0 \\
0\end{array}$ & 1-Public & 2-Durée & $\begin{array}{l}\text { 3-Effec- } \\
\text { tifs }\end{array}$ & 4-Antériorité & $\begin{array}{l}\text { 5-Situations } \\
\text { professionnelles } \\
\text { travaillées }\end{array}$ & $\begin{array}{l}\text { 6-Contenus } \\
\text { interrogés, sur } \\
\text { lesquels porte le } \\
\text { travail réflexif }\end{array}$ & $\begin{array}{l}\text { 7-Modalités } \\
\text { de travail, } \\
\text { outils mobi- } \\
\text { lisés }\end{array}$ & $\begin{array}{l}\text { 8-Progres- } \\
\text { sion péda- } \\
\text { gogique } \\
\text { autour de la } \\
\text { réflexivité }\end{array}$ \\
\hline & $\begin{array}{l}\text { Ingénieur } \\
\text { agro- } \\
\text { nomeen } \\
\text { deuxième } \\
\text { année de } \\
\text { formation } \\
\text { initiale }\end{array}$ & $\begin{array}{l}20 h \text { par } \\
\text { étudiant, } \\
\text { réparties } \\
\text { sur un } \\
\text { semestre }\end{array}$ & $\begin{array}{l}110 \text { étu- } \\
\text { diants par } \\
\text { session }\end{array}$ & $\begin{array}{l}3 \text { ans, } \\
3 \text { sessions } \\
\text { réalisées }\end{array}$ & $\begin{array}{l}\text { Situations mar- } \\
\text { quantes obser- } \\
\text { vées en stage en } \\
\text { entreprise agroindus- } \\
\text { trielle, impliquant au } \\
\text { moins un cadre }\end{array}$ & $\begin{array}{l}\text { Postures de l'ingé- } \\
\text { nieur en tant que } \\
\text { cadre au sein de } \\
\text { l'entreprise }\end{array}$ & $\begin{array}{l}\text { Lectures collec- } \\
\text { tives, partage } \\
\text { autour des } \\
\text { situations mar- } \\
\text { quantes, ateliers } \\
\text { de scénarisa- } \\
\text { tion, séances de } \\
\text { théâtre-forum }\end{array}$ & $\begin{array}{l}\text { Un module } \\
\text { pensé comme la } \\
\text { première étape } \\
\text { d'un dispositif } \\
\text { de formation } \\
\text { plus complet } \\
\text { autour de la } \\
\text { réflexivité }\end{array}$ \\
\hline & $\begin{array}{l}\text { Docto- } \\
\text { rantdu } \\
\text { dpt } \\
\text { Sciences } \\
\text { pour } \\
\text { l'action } \\
\text { et le } \\
\text { dévelop- } \\
\text { pement } \\
\text { (INRA) }\end{array}$ & $\begin{array}{l}1 \text { semaine/ } \\
\text { anpendant } \\
3 \text { ans }\end{array}$ & $\begin{array}{l}15 \text { docto- } \\
\text { rants en } \\
\text { première } \\
\text { année de } \\
\text { thèse, } \\
8 \text { en } \\
\text { deuxième } \\
\text { année, } \\
6 \text { en } \\
\text { troisième } \\
\text { année }\end{array}$ & $\begin{array}{l}14 \text { ans, } \\
14 \text { sessions } \\
\text { réalisées }\end{array}$ & $\begin{array}{l}\text { Vécu professionnel } \\
\text { des doctorants } \\
\text { participant à la } \\
\text { formation = expé- } \\
\text { rience de l'activité de } \\
\text { recherche et trajec- } \\
\text { toire professionnelle } \\
\text { avant la thèse }\end{array}$ & $\begin{array}{l}\text { Pratiques de } \\
\text { recherche (relations } \\
\text { avec les collègues, } \\
\text { les acteurs socio- } \\
\text { économiques...). } \\
\text { Trajectoires d'auto- } \\
\text { nomisation et de } \\
\text { responsabilisation } \\
\text { du chercheur en } \\
\text { tant que producteur } \\
\text { de connaissances } \\
\text { à l'interface entre } \\
\text { science et action }\end{array}$ & $\begin{array}{l}\text { Récits pro- } \\
\text { fessionnels } \\
\text { et ateliers de } \\
\text { co-analyse des } \\
\text { contenus pro- } \\
\text { duits lors des } \\
\text { récits }\end{array}$ & $\begin{array}{l}\text { Chaque docto- } \\
\text { rant peut parti- } \\
\text { ciper } 3 \text { années } \\
\text { de suite à la } \\
\text { formation; ; le } \\
\text { dispositif péda- } \\
\text { gogique est } \\
\text { pensé autour de } \\
\text { cette continuité } \\
\text { et des progres- } \\
\text { sions qu'elle } \\
\text { permet }\end{array}$ \\
\hline & $\begin{array}{l}\text { Stagiaires } \\
\text { d'un } \\
\text { certificat } \\
\text { d'étude } \\
\text { appro- } \\
\text { fondie } \\
\text { en « dia- } \\
\text { gnostic } \\
\text { et projet } \\
\text { de terri- } \\
\text { toire ». } \\
\text { Bac }+3 \text { et } \\
\text { Bac }+5 \text {. }\end{array}$ & $\begin{array}{l}4 \text { semaines } \\
\text { en alter- } \\
\text { nance avec } \\
\text { l'activité } \\
\text { profession- } \\
\text { nelle des } \\
\text { apprenants } \\
\text { sur une } \\
\text { période } \\
\text { de quatre } \\
\text { mois }\end{array}$ & $\begin{array}{l}\text { Une dou- } \\
\text { zaine }\end{array}$ & $\begin{array}{l}2 \text { sessions } \\
\text { réalisées }\end{array}$ & $\begin{array}{l}\text { Vécu professionnel } \\
\text { des participants } \\
\text { durant semaines } \\
\text { dans leur emploi et } \\
\text { semaines en forma- } \\
\text { tion (qui incluent des } \\
\text { actions terrain) }\end{array}$ & $\begin{array}{l}\text { Enjeux relationnels } \\
\text { et partenariaux dans } \\
\text { une dynamique de } \\
\text { projet de territoire. } \\
\text { Attention aux repré- } \\
\text { sentations (a priori, } \\
\text { etc.) qui affectent les } \\
\text { dynamiques indivi- } \\
\text { duelles et collectives }\end{array}$ & $\begin{array}{l}\text { Un dispositif } \\
\text { "réflexivité » } \\
\text { intégré à la } \\
\text { formation : a) } \\
\text { accompagne- } \\
\text { ment individua- } \\
\text { lisé (entretiens } \\
\text { en face à face) } \\
\text { débouchant sur } \\
\text { la production et } \\
\text { la soutenance } \\
\text { orale d'une } \\
\text { note réflexive } \\
\text { individuelle ; } \\
\text { b) temps de } \\
\text { débriefing } \\
\text { collectif. }\end{array}$ & $\begin{array}{l}\text { A adapter à } \\
\text { chaque stagiaire } \\
\text { tutoré selon une } \\
\text { logique } \mathrm{d}^{\prime} \text { indi- } \\
\text { vidualisa-tion }\end{array}$ \\
\hline
\end{tabular}

Tableau 1. Caractéristiques des trois dispositifs de formation analysés.

Ces trois dispositifs, tout en reposant sur des jeux de contraintes (colonnes 2 et 3 du tableau 1) assez différents, ont en commun d'accorder une place importante, voire centrale, au travail de réflexion sur des situations professionnelles réelles vécues par les apprenants, en tant qu'acteurs ou en tant qu'observateurs (colonne 5), permettant d'interroger, de manière concrète et au plus proche des préoccupations des apprenants, des contenus précis à travailler, listés en colonne 6 du tableau 1 . Ces choix pédagogiques ancrent nos pratiques dans une pédagogie de l'alternance. Nous ne cherchons pas à "enseigner la réflexivité », mais à accompagner le renforcement des capacités réflexives. Nos manières de concevoir nos interactions avec les apprenants s'inspirent des travaux de Maëla Paul sur l'accompagnement : prendre les personnes là où elles en sont, laisser advenir et encourager des réflexions et des transformations plutôt que les imposer, s'accrocher à ce qui vient de la personne pour aborder les contenus à travailler, etc. (Paul, 2016). Au-delà de ces points communs, des outils de nature variée sont mis en œuvre dans chacun des trois dispositifs : séances inspirées par les techniques de théâtre-forum (Boal, Mellac, Rigot-Müller, 2004) (D1), utilisation du récit professionnel (Beaujouan et al., 2013) (D2), ou encore entretiens individuels de type rogérien (Rogers, 1968) (D3), pour ne citer que les outils les plus emblématiques de chacun des trois dispositifs. Ces outils sont, à chaque fois, insérés dans un dispositif plus large qui a sa cohérence et ses objectifs propres. En particulier, chaque dispositif s'adresse à un public donné (colonne 1), qui présente des caractéristiques données en termes de capacités réflexives, ce qui a des influences sur les progressions pédagogiques proposées (colonne 8). 
Ainsi, le D1 est pensé comme une «initiation»: c'est la première «brique réflexivité » du cursus, tandis que le D2 se veut plus évolutif : chaque apprenant peut participer trois années de suite à ce même dispositif, les participations successives permettant d'enrichir le travail collectif et, c'est l'hypothèse des concepteurs, de travailler sur un temps plus long les capacités réflexives de chaque participant.

\section{Cadre d'analyse}

Nos premiers échanges ont permis de mettre en lumière le fait que chacun d'entre nous portait des hypothèses, pas forcément explicites, sur les façons d'accompagner le renforcement des capacités réflexives des apprenants, et que ces hypothèses structuraient nos façons de concevoir les progressions pédagogiques. Nous avons souhaité approfondir cette réflexion en rendant plus explicites ces hypothèses et nos manières d'accompagner les apprenants. Pour ce faire, la notion d'étayage a été empruntée à Bruner, définie comme «l'ensemble des interactions d'assistance de l'adulte permettant à l'enfant d'apprendre à organiser ses conduites afin de pouvoir résoudre seul un problème qu'il ne savait pas résoudre au départ» (Bruner, 1998, p. 148). Suivant Vial (2006), nous proposons de «dépersonnaliser» la notion d'étayage, en portant davantage l'attention sur la nature des interactions d'assistance et leur outillage éventuel que sur la personne qui interagit avec l'apprenant : «l'enseignant n'est pas, lui, le médiateur : ce sont les codes, les outils, les signes utilisés pour donner du sens aux actions qui sont les médiateurs du sujet en conflit avec la situation dans laquelle et pour laquelle il utilise ces signes» (Vial, 2006, p.5). Les signes dont parle Vial, que nous appellerons «étais » dans la suite du texte, peuvent être, par exemple, la remémoration de la scène dans un entretien d'explicitation (Vermersch, 1994/2019), les situations et activités organisées par un enseignant (avec notamment la participation des pairs), I'introduction d'objets (par exemple l'imprimerie dans la pédagogie Freinet), etc. Cette attention portée aux «étais» permet de s'interroger sur les modalités précises d'intervention du formateur (montre-t-il? fait-il avec? pose-t-il des questions? fournit-il d'autres ressources? etc.).

\section{Démarche de modélisation}

Nous avons ainsi décidé de caractériser finement ce que nous cherchions à étayer à chaque étape de nos dispositifs, et de préciser les étais mobilisés. Ce travail a été réalisé individuellement par les deux premières auteures de l'article, respectivement pour les dispositifs D1 et D2, plus scénarisés que le D3. II a pris la forme de tableaux présentant en ligne les différentes étapes des dispositifs et en colonne les objectifs pédagogiques ainsi que les étais mobilisés. Les deux conceptrices ont ainsi, dans la colonne "objectifs», formulé des "éléments» qu'elles cherchent à travailler via leurs dispositifs pédagogiques, en vue de renforcer les capacités réflexives d'acteurs sur leurs situations professionnelles. Les formulations suivantes ont par exemple été produites: appréhender une diversité de manières de penser, s'outiller pour analyser, construire du sens, identifier sa propre normativité par rapport à ce qu'est une connaissance scientifique, etc. En dépit de différences dans les formulations proposées par chacune (des éléments formulés de manière très générique, mais relativement imprécise versus des éléments formulés de façon précise, mais très spécifiques des contenus travaillés dans le dispositif), nous avons constaté qu'un certain nombre d'axes se recoupaient, qui faisaient également écho à l'expérience du troisième auteur, et faisaient sens pour la quatrième auteure. Nous avons cherché à formaliser ces axes communs, en nous appuyant sur un travail de questionnement entre pairs, visant à faire préciser autant que possible nos propos et à confronter nos différentes formulations à nos expériences de formateurs, incluant les trois dispositifs tels qu'initialement conçus, mais ne s'y limitant pas. C'est ainsi que la dernière version de ce processus itératif, présentée à la figure 2, fait apparaître la réflexivité comme composée de cinq dimensions sur lesquelles nos expériences montrent, nous l'illustrons dans la section suivante, qu'il est possible de travailler d'un point de vue pédagogique.

\section{Une grille-repères pour la conception de dispositifs visant à renforcer les capacités réflexives d'ac- teurs sur leurs situations professionnelles}

Pour réfléchir à la conception de dispositifs pédagogiques visant à accompagner le renforcement des capacités réflexives d'acteurs sur leurs situations professionnelles, à des fins de développement, nous proposons d'envisager la réflexivité sous la forme de cinq dimensions interreliées, formulées à la figure 2 de manière volontairement simple. 
a- J'ai une certaine conscience qu'en situation professionnelle, tout le monde n'agit, ne réfléchit, ne ressent pas les choses de la même façon

b- J'ai une certaine conscience d'être un sujet à part entière : j'agis, je réfléchis et je ressens à ma façon, et cela peut avoir un impact sur le cours des choses

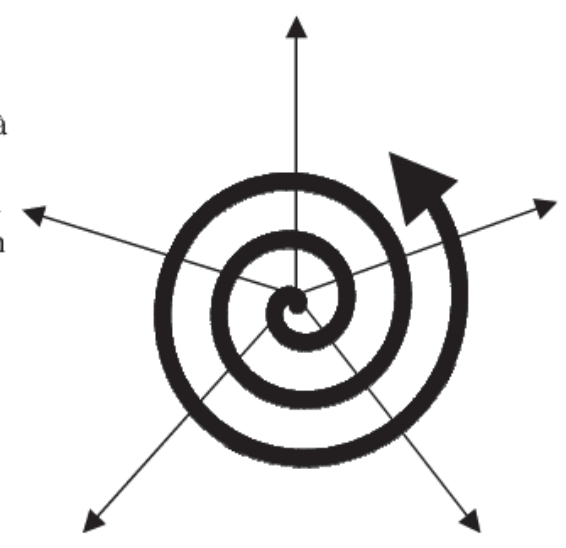

e- J'identifie, je remets en question et je fais évoluer mes cadres d'analyse des situations professionnelles c- J'analyse des situations professionnelles me concernant et/ou concernant autrui : je mets des mots sur ce qui se joue, sur ce que ça dit de moi/des autres/ du contexte professionnel et de son jeu de contraintes d- Dans des situations professionnelles données, j'envisage et je mets en œuvre d'autres manières d'agir : plus adaptées / efficaces / pertinentes / mettant davantage en adéquation valeurs et pratiques / respectant mieux le jeu de contraintes qui m'est imposé, etc.

Figure 2. Cinq dimensions de la réflexivité qu'il est possible de travailler d'un point de vue pédagogique.

Ces cinq dimensions renvoient à cinq aspects de la réflexivité qu'il est possible de travailler directement avec des apprenants pour les accompagner dans le renforcement de leurs capacités réflexives sur leurs situations de travail. Elles sont interconnectées et non hiérarchisées : travailler sur une dimension donnée peut aider à en développer une autre, comme nous l'illustrerons plus bas, selon des cheminements qui n'ont rien de préétabli. Chacune de ces dimensions proposées est à considérer comme une variable continue plutôt qu'une variable binaire du type acquis/non acquis.

Ces dimensions renvoient en effet à des habitudes cognitives et des savoir-faire qu'il est selon nous toujours possible de renforcer. Ainsi, si une personne donnée est relativement accoutumée à une certaine pratique réflexive dans un milieu professionnel donné, la dimension a peut, par exemple, lui paraître triviale. En revanche, face à des personnes culturellement très différentes d'elle, elle peut se trouver démunie pour appréhender leurs manières d'être et d'agir, indiquant par-là l'existence de marges de progression la concernant sur la dimension a.

Dans les sections suivantes, nous discutons, pour chaque dimension: (i) sa pertinence; (ii) des exemples d'étais mobilisables pour la travailler; (iii) des illustrations, puisées dans nos expériences de formateurs, de résultats incidents d'un tel travail pour les apprenants. 


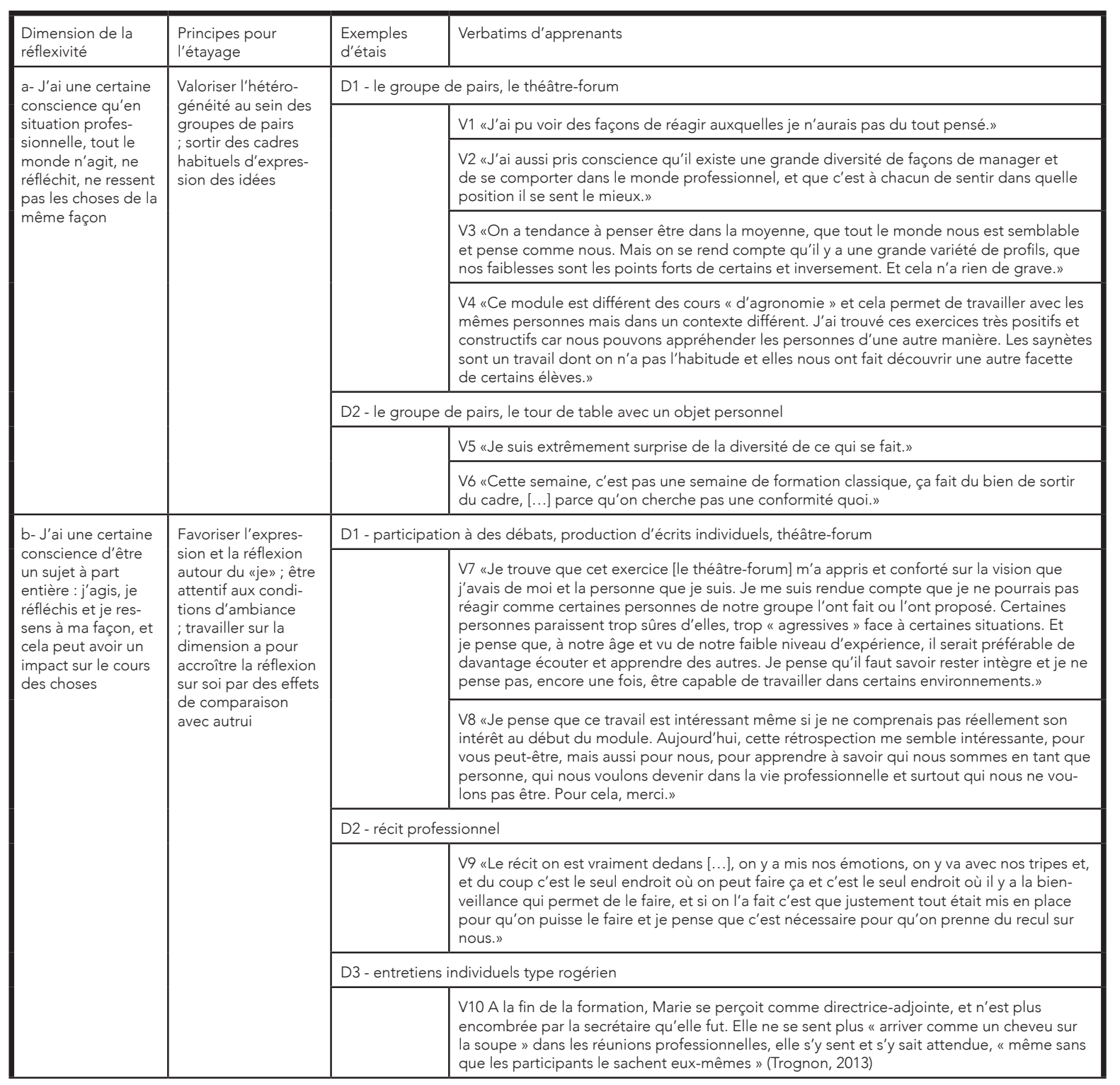




\begin{tabular}{|c|c|c|}
\hline \multirow{7}{*}{$\begin{array}{l}\text { c- J'analyse des } \\
\text { situations pro- } \\
\text { fessionnelles me } \\
\text { concernant et/ou } \\
\text { concernant autrui } \\
\text { : je mets des mots } \\
\text { sur ce qui se joue, } \\
\text { sur ce que ça dit } \\
\text { de moi, des autres, } \\
\text { du contexte profes- } \\
\text { sionnel et de son } \\
\text { jeu de contraintes }\end{array}$} & \multirow{7}{*}{$\begin{array}{l}\text { Proposer des } \\
\text { outils et méthodes } \\
\text { d'analyse à la fois } \\
\text { suffisamment spé- } \\
\text { cifiques de l'objet } \\
\text { traité et suffisam- } \\
\text { ment génériques } \\
\text {; tenir compte des } \\
\text { capacités d'analyse } \\
\text { des personnes en } \\
\text { formation ; notion } \\
\text { de bricolage et de } \\
\text { réadaptation per- } \\
\text { manente des outils } \\
\text { d'analyse à faire } \\
\text { travailler }\end{array}$} & $\begin{array}{l}\text { D1 - des débriefings qui sensibilisent sur les différences entre description, interprétation et jugement ; } \\
\text { concepts de genre et style de Clot et Faïta (2000) }\end{array}$ \\
\hline & & $\begin{array}{l}\text { V11 «La méthode utilisée pour faire la distinction entre décrire, interpréter et ju- } \\
\text { ger était adaptée à l'exercice. Le débat ainsi constructif a permis de faire avancer la situa- } \\
\text { tion de départ.» }\end{array}$ \\
\hline & & $\begin{array}{l}\text { D2 - concepts de réductionnisme, holisme, positivisme, constructivisme embarqués dans des outils d'analyse } \\
\text { mobilisés et ré-adaptés session après session }\end{array}$ \\
\hline & & $\begin{array}{l}\text { V12 «J'ai l'impression que ces outils on a quand même pu bien se les approprier parce } \\
\text { qu'on a travaillé dessus et je pense que j'ai commencé à réfléchir [...] je sais pas si j'aurai } \\
\text { l'occasion ou pas de revenir, mais même si je reviens pas, je sais que toujours ces outils } \\
\text { resteront jusqu'à la rédaction de la thèse dans ma tête et que ça m'a vraiment appris beau- } \\
\text { coup de choses.» }\end{array}$ \\
\hline & & $\begin{array}{l}\text { V13 «J'ai l'impression de mieux comprendre pourquoi j'ai ces problèmes-là et de mettre des } \\
\text { mots sur la nature des doutes que j'ai par rapport à mon travail.» }\end{array}$ \\
\hline & & $\begin{array}{l}\text { V14 «J'avais pas conscience que les questions qu'on avait aujourd'hui ça faisait partie du } \\
\text { travail, j'avais l'impression que c'était des trucs dans ma tête seulement - ça fait plaisir de } \\
\text { voir que c'est des vraies questions, qu'elles ont un sens, que je peux me les poser et qu'il y } \\
\text { a une réflexion derrière sur laquelle je peux me reposer.» }\end{array}$ \\
\hline & & $\begin{array}{l}\text { V15 «J'ai pas envie qu'on m'apprenne à être réflexive, parce que je pense que je suis déjà } \\
\text { réflexive, j'ai envie qu'on m'aide à gérer ma réflexivité, parce que parfois elle part dans tous } \\
\text { les sens, elle peut être même autodestructrice [...] enfin voilà, et je trouve que c'est ce qu'on } \\
\text { a fait, donc je suis contente.» }\end{array}$ \\
\hline \multirow{5}{*}{$\begin{array}{l}\text { d- Dans des situa- } \\
\text { tions profession- } \\
\text { nelles données, } \\
\text { j'envisage et je } \\
\text { mets en œuvre } \\
\text { d'autres manières } \\
\text { d'agir : plus adap- } \\
\text { tées, efficaces, } \\
\text { pertinentes, etc. }\end{array}$} & \multirow{5}{*}{$\begin{array}{l}\text { Inciter les personnes } \\
\text { à formuler des pistes } \\
\text { d'action mobili- } \\
\text { sables dans leur } \\
\text { pratique ; travailler à } \\
\text { la constitution d'un } \\
\text { "catalogue person- } \\
\text { nel»; encourager la } \\
\text { réflexion de groupe } \\
\text { autour de situations- } \\
\text { problème }\end{array}$} & D1 - théâtre-forum \\
\hline & & $\begin{array}{l}\text { V16 «Si un problème comme ceux que nous avons pu jouer nous arrive dans notre futur } \\
\text { poste, nous serons plus préparés à faire face à ces contraintes. Nous avons songé à des } \\
\text { solutions qui ne nous seraient pas venues à l'esprit si nous étions tout seul face à cela. } \\
\text { Même si cela apparaît comme un exercice tout simple et «basique », nous avons envisagé } \\
\text { des solutions et nous avons donc gagné de l'expérience de manière implicite.» }\end{array}$ \\
\hline & & $\begin{array}{l}\text { V17 «Le fait d'être plusieurs dans un groupe pour trouver des solutions aux problèmes } \\
\text { posés semble être un moyen efficace de réfléchir.» }\end{array}$ \\
\hline & & D3 - jeux de rôle \\
\hline & & $\begin{array}{l}\text { V18 «Souvenir positif de cet exercice [...]. Rapidement, si je dois en retenir quelque chose } \\
\text { pour ma pratique, il me paraît primordial, dans le cadre de projets [...], de développer une } \\
\text { capacité d'écoute pour comprendre les positionnements et revendications de chacun, et pouvoir } \\
\text { proposer des actions qui peuvent être entendues et acceptées par les différents acteurs autour de la } \\
\text { table (permettant de ne pas amener le projet droit dans le mur!)» }\end{array}$ \\
\hline \multirow{4}{*}{$\begin{array}{l}\text { e- J'identifie, je } \\
\text { remets en ques- } \\
\text { tion, je fais évoluer } \\
\text { et j'enrichis mes } \\
\text { cadres d'analyse } \\
\text { des situations } \\
\text { professionnelles }\end{array}$} & \multirow{4}{*}{$\begin{array}{l}\text { Faciliter un travail de } \\
\text { prise de conscience } \\
\text { des cadres d'ana- } \\
\text { lyse spontanément } \\
\text { mobilisés; confron- } \\
\text { ter les apprenants à } \\
\text { des cadres d'analyse } \\
\text { inédits pour eux } \\
\text {; sensibiliser les } \\
\text { apprenants aux } \\
\text { intérêts de réinter- } \\
\text { roger et renouveler } \\
\text { ses cadres d'analyse } \\
\text { en permanence }\end{array}$} & D1 - vidéo vulgarisant le principe des cités de Boltanski et Thévenot (1991) \\
\hline & & $\begin{array}{l}\text { V19 «Je me suis [...] rendu compte lors du théâtre-forum que j'aime essayer de résoudre } \\
\text { des conflits. Cet intérêt s'est renforcé quand vous nous avez rapidement présenté la théorie } \\
\text { des cités [...]. Je trouve que cette théorie est intéressante car elle insinue que des conflits } \\
\text { peuvent être dus au fait que l'on n'accorde pas la même importance à certaines valeurs et } \\
\text { que l'on n'utilise pas le même vocabulaire. Ainsi, une façon de résoudre un conflit peut être } \\
\text { de trouver un vocabulaire et des valeurs communes qui vont permettre aux personnes de se } \\
\text { comprendre. Avant cela, je m'intéressais déjà aux conflits (j'aime en effet me renseigner sur } \\
\text { la géopolitique) et le module X avait aussi confirmé mon intérêt dans l'étude de ces phéno- } \\
\text { mènes. [...] Ainsi, peut être que des postes de médiation pourraient me convenir aussi ? » }\end{array}$ \\
\hline & & D3 - entretiens individuels type rogérien \\
\hline & & $\begin{array}{l}\text { V20 «Le cours de Madame X [politologue] m'a ouvert les yeux. Les PNR, je n'arrivais pas à } \\
\text { les voir comme des partenaires. Ils m'agacent avec toutes leurs contraintes. Je n'avais pas } \\
\text { perçu qu'ils jouent un rôle important et que leur position s'explique et se justifie [...]. C'est } \\
\text { sûr, maintenant, je ne les verrai plus de la même manière.» (Trognon, 2013) }\end{array}$ \\
\hline
\end{tabular}

Tableau 2. Principes pour l'étayage, exemples d'étais mobilisés dans nos trois dispositifs D1, D2 et D3, et exemples de verbatims d'apprenants pour chacune des cinq dimensions de la réflexivité.

\section{Dimension a : avoir conscience de la diversité des façons d'agir, de réfléchir et de ressentir}

Dès leurs premières expériences pédagogiques autour de la réflexivité, les co-auteurs partagent un constat : pour beaucoup d'étudiants ingénieurs, de doctorants ou d'agents de développement territorial en formation continue, l'intérêt d'entrer dans un exercice réflexif n'est pas toujours perçu. 
Nous avons mis en lien ce constat avec le fait que, par exemple, les étudiants-ingénieurs avec lesquels nous travaillons sont souvent dans l'attente que nous leur délivrions "les bonnes manières de faire», à savoir des recettes adaptables à toute la gamme des situations professionnelles auxquelles un jeune ingénieur agronome est susceptible de se trouver confronté («ll faudrait faire intervenir des professionnels [...] qui peuvent nous former dans les situations concrètes de l'entreprise à adopter les bonnes manières ", nous écrivent les étudiants ingénieurs en rentrant de stage, dans le cadre d'un dispositif d'évaluation des enseignements par les étudiants). Ces étudiants ont souvent un point de vue très normatif sur ce qu'ils observent et entendent en stage. "Ce n'est pas professionnel», "ça m'a choqué, ça ne se fait pas», s'emportent-ils souvent, sans pour autant être en mesure d'expliciter en quoi cela «ne se fait pas» ni à quels critères de "professionnalité » ils font référence. Dans une telle quête de la «bonne manière de faire » et d'une approbation quasi unanime de ces «bonnes manières", non seulement il y a peu de place pour l'appréhension de la diversité, mais il peut même y avoir des résistances à son acceptation. À notre sens, ce phénomène peut être relié, notamment, à l'importance donnée aux sciences dites «dures» dans nos formations, et à la croyance selon laquelle ces sciences sont capables de «dire le vrai», un «vrai » univoque et totalement dépourvu d'ambiguïté, susceptible de dicter des conduites à tenir. Ces questions sont particulièrement sensibles avec le public des doctorants (D2), pour qui une telle croyance peut parfois constituer un élément d'engagement professionnel assez fort. L'idée selon laquelle il n'y aurait qu'une seule bonne façon de faire de la science et de définir ce qui est scientifique et ce qui ne l'est pas reste assez répandue au sein de ce public. Les doctorants sont pourtant confrontés dans leurs unités de recherche et dans leur pratique scientifique à une diversité plus importante et à des controverses autour de ces points, mais ces dernières ne sont pas toujours explicites, et elles sont parfois maladroitement interprétées en termes de différends entre personnes. Ce type d'observation se retrouve aussi en formation continue chez des personnes dont les représentations ont eu tendance à se raidir.

Face à de telles problématiques, il nous a paru pertinent de travailler une certaine ouverture à la diversité et à la pluralité des représentations et des manières d'agir, de réfléchir et de ressentir et de formuler explicitement ces aspects-là comme une dimension à part entière du travail réflexif. Pour réaliser un tel travail, nous mobilisons plusieurs types d'étais. En premier lieu, s'appuyer sur le "groupe de pairs» nous paraît particulièrement adapté. Notre hypothèse est la suivante : faire émerger des éléments de diversité à l'intérieur d'un collectif qui paraît pourtant homogène à ses membres peut constituer une expérience légèrement «bousculante» pour les membres du groupe, de nature à enclencher un cheminement allant dans le sens d'une plus grande ouverture à la diversité. Nous voyons ainsi un véritable enjeu à rechercher et à mettre en valeur l'hétérogénéité au sein de l'homogénéité apparente du groupe de pairs. Le mode de constitution des groupes de travail dans le D2 traduit bien cet état d'esprit : les porteurs du dispositif cherchent autant que possible à mixer les appartenances disciplinaires et les types de sujets de recherche et de rapports à l'action portés par les doctorants. Une fois l'hétérogénéité ainsi savamment distillée, reste à savoir comment la faire s'exprimer et, en quelque sorte, "s'épanouir» au sein des groupes de travail. Sur ce point, il nous semble utile de sortir des cadres d'expression des idées auxquels les participants sont accoutumés (présentation de diaporamas préparés en amont, argumentation de problématiques scientifiques...), qui tendent à homogénéiser les propos et la façon de les présenter. Cela peut passer, avec nos publics, par la centration sur le vécu individuel de chacun, ou encore des aspects de scénarisation (au sens théâtral du terme) d'expériences : de la mise en espace et en action au-delà de la simple mise en mots. Ainsi, dans le D2, un exercice complémentaire au traditionnel tour de table de présentation est proposé : les doctorants en première année de thèse sont invités à donner à voir et à exprimer leur motivation à s'engager dans un travail de thèse à partir d'un objet qu'ils ont choisi au préalable et qu'ils apportent avec eux et commentent en formation. Cela permet de donner à voir une diversité de rapports à l'exercice de thèse et à la thématique de la thèse. Dans le D1, les étudiants doivent, en groupe, mettre en scène et jouer une situation réelle observée en stage, mettant en jeu le comportement d'un cadre au sein de l'entreprise, qu'ils jugent non satisfaisante. Ensuite, suivant certains principes du théâtre-forum (Boal et al., 2004), les étudiants spectateurs sont invités à imaginer d'autres comportements possibles pour les personnages de la scène ainsi jouée et à les donner à voir, non pas en expliquant comment tel personnage aurait pu se comporter autrement, mais en incarnant directement cette proposition sur scène. Une diversité de manières de voir, de penser et d'agir en situation professionnelle est ainsi littéralement donnée en spectacle par les étudiants eux-mêmes.

À la fin du D1, les étudiants doivent chacun rédiger une note réflexive, portant notamment sur leurs évolutions et leurs questionnements durant le module. Certains témoignages ainsi obtenus illustrent bien les évolutions qui ont pu avoir lieu en termes d'ouverture à la diversité dans les manières d'agir, de réfléchir et de ressentir (voir les verbatims V1 et V2 du tableau 2). Un verbatim illustre particulièrement bien notre idée de «faire émerger l'hétérogénéité dans I'homogénéité » (V3). Enfin, un dernier verbatim met bien en évidence l'intérêt de proposer des modalités de travail inhabituelles pour mieux s'ouvrir à la diversité et à la complexité d'autrui (V4). Concernant le D2, un tour de table de débriefing a lieu en fin de formation, dont le contenu a été retranscrit en 2016 et en 2017. On retrouve dans ce matériau des idées similaires à celles énoncées pour le dispositif 1 (voir V5 et V6). 


\section{Dimension b : avoir conscience d'être un sujet à part entière}

Cette dimension est très liée à la précédente, et les deux dimensions peuvent se travailler ensemble. On conçoit en effet aisément qu'une meilleure perception de la diversité des manières d'agir, de réfléchir et de ressentir d'autrui peut conduire, par des effets de comparaison, à une connaissance plus fine de soi. Toutefois, nous avons fait le choix de maintenir dans notre modèle une dimension consacrée spécifiquement à cette idée du «sujet». C'est une dimension spécifique qui nous paraît intéressante à travailler pour tous, et cette idée s'illustre particulièrement bien dans le cas des doctorants. Pour ces derniers, il peut en effet exister une croyance dans la neutralité axiologique comme caractéristique de qualité de la science. Dans une telle science dite «objective», le sujet-chercheur n'aurait pas son mot à dire dans le choix des questions et des façons de les traiter; ce sont les disciplines et les «lois» de la recherche qui s'imposeraient à lui. Cette idée que le sujet-chercheur n'aurait pas vraiment sa place représente non seulement une vision tronquée de la réalité, mais elle est également susceptible de générer des tensions chez les personnes concernées, particulièrement quand les thématiques de recherche sont également des questions socialement vives : la préservation de l'environnement, la production agricole, l'alimentation, la gouvernance territoriale, etc.

Pour sortir de cette impasse, il est, à nos yeux, indispensable que le dispositif permette de replacer le sujet au cœur des réflexions, et de reconnaître son importance dans la pratique scientifique, et, plus largement, dans toute pratique professionnelle. Les concepteurs du D2 ont ainsi choisi le récit professionnel (Beaujouan et al., 2013) comme un pilier de leur dispositif de formation. Il s'agit de donner la parole au sujet-chercheur, qui produit, devant un groupe de pairs et un binôme d'animateursformateurs, un récit à la première personne à partir d'une question très ouverte («raconte nous ton expérience de thèse »), pendant un temps assez long (1 h30), avec des questions de relance qui soutiennent l'explicitation de ce qui a été fait, avec qui et pourquoi, sans approfondir ni critiquer les raisonnements scientifiques. Cet exercice vise "le pas de côté » par rapport à la norme de l'échange scientifique entre pairs, pour accéder à l'activité du sujet dans les divers aspects de son engagement personnel. Cette pratique du récit professionnel est sans doute, parmi les étais que nous mobilisons, celle qui permet de travailler le plus en profondeur la dimension b de notre modèle. Toutefois, d'autres étais, de mise en place plus simple, sont tout-à-fait mobilisables pour accompagner les apprenants dans le renforcement de certains aspects de prise de conscience de soi. Il peut s'agir, par exemple, de jeu de rôle, ou tout simplement d'inciter les personnes à formuler et partager un point de vue individuel, les faire participer à un débat, leur suggérer de coucher sur le papier des réflexions individuelles... ou toute autre pratique qui donne de la place à l'expression du «je». Les entretiens individuels de type rogérien (Rogers, 1968), par l'effet miroir qu'ils permettent, s'avèrent également être des outils intéressants pour renforcer certains aspects de prise de conscience de soi.

Ainsi, dans le D3, Marie, stagiaire en formation continue, change progressivement son regard sur elle-même, au gré d'une série d'entretiens individuels (V10). S'agissant du travail avec les doctorants (D2), une participante souligne la pertinence du dispositif mis en place, comprenant, entre autres, l'exercice du récit professionnel évoqué ci-dessus, mais aussi un certain nombre de conditions d'ambiance, pour arriver à engager une authentique réflexion sur soi (V9). Dans le cadre du D1, une étudiante souligne, dans son écrit réflexif, l'idée que la comparaison avec les autres (dimension a) peut aider à renforcer des aspects de connaissance de soi (dimension b) (V7). Elle conclut plus loin, en s'adressant à ses «lecteurs-formateurs», sur l'intérêt de l'étai "production d'une note réflexive individuelle» (V8).

\section{Dimension c : analyser des situations professionnelles}

Cette dimension est au cœur des processus cognitifs auxquels on s'attend dès lors qu'on parle de réflexivité : il s'agit de prendre du recul et de produire un discours sur des situations professionnelles vécues, à des fins de développement. Nos expériences respectives montrent que cette capacité à analyser des situations professionnelles et à en tirer des enseignements ne va pas toujours de soi. Même lorsqu'ils ne présentent a priori pas de réticence par rapport à l'exercice réflexif, il ne suffit en général pas de demander aux apprenants d'analyser des situations professionnelles pour que ces derniers produisent une réflexion de nature à alimenter une ré-interrogation de leurs pratiques et/ou du contexte professionnel dans lequel ils exercent. Des grilles d'analyse ou tout au moins des méthodologies d'analyse pertinentes sont nécessaires pour cela.

L'étayage à mettre en place pour accompagner les apprenants dans l'apprentissage du travail d'analyse de situations professionnelles dépend en premier lieu des objets travaillés (cf. colonne 6 du tableau 1). Si on veut renforcer la consistance et la pertinence de l'analyse, il est nécessaire de se doter de grilles suffisamment spécifiques des objets sur lesquels portera la réflexivité. À défaut, le risque est grand de produire un discours superficiel ayant peu de chance de véritablement faire sens pour la personne qui l'énonce. En revanche, une moindre spécificité des grilles ou méthodologies d'analyse facilitera leur remobilisation et pourra inspirer à l'apprenant d'autres analyses dans d'autres contextes. 
Outre cet arbitrage entre spécificité et généricité des méthodes et grilles d'analyse, il convient de s'interroger sur les connaissances et savoir-faire préexistants ou non chez les apprenants. Si on souhaite, comme c'est le cas pour le D1, travailler sur l'objet «postures de l'ingénieur en tant que cadre au sein de l'entreprise», en insistant sur la diversité des postures individuelles possibles et sur la nécessaire prise en compte des contextes organisationnels d'exercice de l'activité, les concepts de genre et style de Clot et Faïta (2000) apparaissent comme un étai pertinent pour la réflexion et l'analyse. En revanche, nous l'avons éprouvé, faire lire Clot et Faïta (2000) directement dans le texte à des étudiants peu familiers des écrits scientifiques en sciences humaines et sociales ne facilitera le travail d'analyse que pour une très petite poignée d'étudiants. II convient donc d'adapter ses étais aux connaissances et savoir-faire des personnes à accompagner, selon une logique itérative de «bricolage » et de réadaptation année après année. Au fil des années, il a ainsi paru pertinent aux formateurs du D1 et du D3 d'apporter un étayage sur des méthodologies d'analyse relativement «basiques», par exemple en sensibilisant les apprenants aux différences entre description, interprétation et jugement. Dans le D2, la réflexivité doit porter notamment sur les pratiques de recherche des doctorants. Les animateurs de ce dispositif ont fait le choix de travailler, notamment, des questions d'ordre épistémologique. II a ainsi pu être question dans le dispositif de notions comme le réductionnisme, I'holisme, le positivisme ou encore le constructivisme. L'enjeu n'était pas que les doctorants maîtrisent toute l'histoire de la pensée épistémologique et tous les tenants et aboutissants de chacun de ces concepts; I'idée était plutôt d'utiliser cet ensemble de concepts comme un outil heuristique pour faire réfléchir les doctorants à la variété des positionnements épistémologiques possibles et à leur propre positionnement. Les modalités d'utilisation de cet étayage conceptuel ont beaucoup évolué, par ajustements successifs, au cours des 14 années d'expérience du D2, donnant des parts variables dans le temps à des modalités de travail comme les présentations magistrales, la mise en pratique au sein de groupes de travail, les lectures d'articles scientifiques, le débat avec des personnes expertes. La manière de présenter et de nommer les concepts a également évolué, certaines dénominations ayant été abandonnées au profit d'autres, moins génératrices de tensions et/ou plus pertinentes par rapport aux enjeux réels des doctorants.

Un tel travail de «bricolage» d'étais au service d'un renforcement des capacités d'analyse des situations professionnelles semble porter ses fruits puisque nombreux sont les apprenants qui soulignent, d'une manière ou d'une autre, l'intérêt de la sensibilisation méthodologique et/ou des outils d'analyse que nous leur proposons dans le cadre de nos trois dispositifs. Ainsi, les participants au D1 et au D3 mettent en avant, dans leurs notes réflexives, certains apports méthodologiques qui peuvent les aider à structurer leur réflexion sur des situations professionnelles (V11, tableau 3). Quant aux doctorants du D2, ils sont nombreux à souligner l'intérêt des outils d'analyse proposés, certains évoquant même la possibilité de les remobiliser en autonomie (V12). Pour eux, ces outils peuvent être au service d'une meilleure compréhension de sa situation propre (V13), d'une légitimation du fait de se poser des questions sur ses pratiques et postures de recherche (V14), ou ils peuvent être perçus comme un guide permettant de «canaliser» sa réflexivité et de la rendre «constructive» (V15).

\section{Dimension $d$ : se projeter dans d'autres manières d'agir}

Au-delà de l'analyse des situations professionnelles, la réflexivité renvoie également à une capacité de ré-interrogation de ses actions et de projection dans l'action. Cette dimension serait particulièrement intéressante à travailler avec des personnes à I'aise avec un travail de conceptualisation, et avec l'idée de parler de soi, mais ayant des difficultés à transformer cette réflexion en action. N'ayant pas perçu de difficulté critique de nos apprenants sur cette dimension, nous n'avons pas conçu ou testé d'étais spécifiques. En revanche, l'outil théâtre-forum pourrait représenter, par exemple, un étai intéressant. À travers l'usage du théâtre-forum dans le D1, notre ambition première était de sensibiliser les étudiants à la diversité des manières d'agir, de réfléchir et de ressentir en milieu professionnel, mais on peut tout-à-fait envisager cet outil comme un étai intéressant pour faciliter un travail de projection dans l'action, ce que ne manquent pas de souligner certains étudiants dans leurs écrits réflexifs. Ainsi, un étudiant nous explique que l'expérience vécue via le théâtre-forum pourrait lui être directement utile dans son futur professionnel (V16). II tire de cette réflexion une autre conclusion, sous la forme d'une règle qui pourrait structurer des actions à venir (V17). II est intéressant de noter que des jeux de rôle, mobilisés par le concepteur du D3, peuvent susciter des projections similaires (V18). On pourrait imaginer bien sûr de nombreux autres étais pour travailler sur cette dimension, valorisant par exemple le fait de devoir énoncer devant d'autres personnes ou coucher sur le papier des pistes concrètes d'action, ou encore, comme dans le cas du théâtre-forum, valorisant la réflexion en groupe. Plus simplement, on peut aussi imaginer, comme le font les concepteurs du D2, que mieux analyser les situations professionnelles (dimension c) peut également être vu comme une manière de faire émerger et pratiquer de nouvelles manières d'agir.

\section{Dimension e : questionner ses cadres d'analyse des situations professionnelles}

Cette dimension est très liée à la dimension c, puisque ces deux dimensions portent sur les cadres et méthodologies d'analyse des situations professionnelles. 
Alors que la dimension c renvoyait à l'idée d'apprendre à analyser des situations professionnelles en mobilisant des cadres d'analyse données, souvent suggérés par les formateurs, l'idée nouvelle, à travers cette dimension e, est de faire porter son attention sur les cadres d'analyse qu'on mobilise le plus fréquemment, sans même y réfléchir : quels sont les cadres d'analyse que je mobilise le plus spontanément?

En quoi sont-ils heuristiques ou au contraire limitants pour une meilleure appréhension des situations professionnelles qui me concernent? Ne pourrais-je pas les faire évoluer, voire les enrichir par de nouveaux cadres d'analyse? Cette dimension est très intéressante à travailler avec des personnes prisonnières d'une représentation bloquante de leur environnement professionnel, par exemple lorsqu'elles analysent leur environnement professionnel et leurs partenaires potentiels selon une grille «ennemis vs amis» : "les PNR [Parcs Naturels Régionaux] c'est l'ennemi », explique Aline lors de ses premières rencontres avec son enseignant-tuteur dans le cadre du D3 (Trognon, 2013). Au-delà, prendre I'habitude de réinterroger ses cadres d'analyse, d'adopter de nouveaux points de vue et de renouveler sa réflexion en la nourrissant de manières d'analyser inédites pour soi est une disposition tout-à-fait intéressante à travailler dans l'optique d'une pratique réflexive autonome et assumée, à des fins de développement professionnel et personnel.

Pour travailler cette dimension avec des apprenants, il peut être intéressant de faciliter un travail de prise de conscience des cadres d'analyse spontanément mobilisés et/ou de confronter les apprenants à d'autres cadres d'analyse possibles, sans leur imposer de les mobiliser. Les entretiens individuels de type rogérien (Rogers, 1968) ou, plus largement, un travail de nature sémantique mené aussi fréquemment que possible constituent un outil de choix pour pointer des représentations bloquantes et lever certaines difficultés. Ainsi, dans le cadre du D3, lorsqu'Aline argumente dans un quasi-monologue que «les PNR [Parcs Naturels Régionaux], c'est l'ennemi!», son enseignant-tuteur lui renvoie simplement une question : «Ennemi?», et l'amène progressivement à préciser, avec diverses techniques de relance et de reformulation, qui ou quoi se cache derrière cet étiquetage et sa propre attitude dans ses relations avec eux, amenant la personne à remettre en question son cadre d'analyse initial (Trognon, 2013). Dans le D1, à l'issue des séances de théâtre-forum, les étudiants visionnent une courte séquence vidéo (Gardet, 2016) présentant de façon vulgarisée le principe des cités de Boltanski et Thévenot (1991). L'animatrice les invite ensuite à commenter rapidement ce qui s'est joué lors des séances de théâtre-forum : de quelle(s) cité(s) relevaient majoritairement les argumentaires déployés lors des saynètes?

Année après année, les étudiants ingénieurs relèvent de fortes proximités entre la cité industrielle et la cité domestique d'une part et leurs façons d'appréhender le monde d'autre part. Avec des intensités variables en fonction des années et des groupes, ils avouent parfois aussi se sentir proches de la cité marchande et/ou de la cité civique, et restent en général distants vis-à-vis de la cité de l'opinion et de la cité inspirée. L'idée de cet exercice est bien de les sensibiliser à d'autres façons d'appréhender le monde que les leurs, et à l'existence de grilles d'analyse permettant de rendre compte de cette diversité et des tensions qui s'expriment en son sein. Dans tous les cas, il revient bien aux apprenants de s'emparer ou non de ces cadres d'analyse, le travail d'étayage consistant «simplement» à révéler leur existence aux apprenants dans des conditions et à des moments que les formateurs jugent propices à ce type de réflexion. Enfin, dans le D2, le simple fait de rendre visibles les ajustements permanents des cadres d'analyse réalisés par l'équipe de formateurs permet de sensibiliser les doctorants à la pertinence de réinterroger et d'enrichir ses cadres d'analyse en permanence.

Certains témoignages d'apprenants montrent bien qu'un tel étayage peut faire sens pour eux et leur ouvrir de nouvelles perspectives. Ainsi en va-t-il pour Aline qui, un mois après le début de sa formation (D3), se présente radieuse face à son enseignant-tuteur, lui expliquant qu'elle ne considère plus les PNR comme des ennemis (V20). Ici, soulignons l'importance de tout un dispositif pédagogique fait d'interactions entre approches individuelles et collectives partagées à l'échelle d'une équipe pédagogique. Nous disposons également de témoignages relatant certaines prises de conscience ou réaffirmations, étayées par les outils que nous avons mis en place. Ainsi un étudiant du D1 s'empare-t-il véritablement de ce qu'il comprend de la théorie des cités de Boltanski et Thévenot (1991) pour produire une réflexion en lien avec son projet professionnel (V19). À travers ce témoignage, l'étudiant combine au moins trois dimensions de la réflexivité telle que nous la décrivons : il fait la preuve d'une capacité à enrichir ses cadres d'analyse (ici des conflits) à partir d'un élément nouveau dont il s'empare (la théorie des cités) (dimension e), au service d'une réflexion qui débouche sur des perspectives d'action (ici des idées pour résoudre des conflits) (dimension d) et le conduit à réinterroger des aspects de son projet professionnel et personnel en construction (et pourquoi pas la médiation?) (dimension b). On peut imaginer qu'un tel travail cognitif, à nos yeux emblématique de ce qu'on peut attendre d'une démarche de réflexivité, et facilité ici par tout un ensemble d'étais mis en place dans le cadre du D1, pourrait tout-à-fait être reproduit dans d'autres types de contextes par l'étudiant en question, avec un étayage peut-être moins important. C'est en tout cas le sens de nos propositions sur l'étayage de processus de renforcement des capacités réflexives d'acteurs sur leurs situations professionnelles. 


\section{Discussion conclusive}

\section{Positionnement de notre grille par rapport à la littérature}

Les grilles présentes dans la littérature autour de la notion de réflexivité à des fins de développement professionnel (Van Manen, 1977; Hatton et Smith, 1995; Sparks-Langer, Simmons, Pasch, Colton, Starko, 1990; Jorro, 2005; Buysse et Vanhulle, 2009) permettent surtout, à notre connaissance, de caractériser des niveaux atteints ou non par des apprenants. Notre grille est originale dans la mesure où, contrairement aux grilles précédemment citées, elle n'a pas de finalité évaluative, mais vise à aider les enseignants praticiens à concevoir des dispositifs permettant de renforcer les capacités réflexives des apprenants. Précisons ici que notre propos n'est pas de dicter les «bonnes manières de faire » en matière d'accompagnement du renforcement des capacités réflexives. Les illustrations proposées dans cet article ont, bien sûr, été choisies pour mettre en évidence les aspects les plus concluants de notre travail pédagogique. Dans la pratique, tout ne fonctionne pas avec tous les apprenants et certaines actions pédagogiques mises en œuvre restent malheureusement sans effet apparent sur certains apprenants. De même, les co-auteurs ne sont pas tous à l'aise avec tous les étais évoqués : chacun a ses préférences, ses outils et démarches avec lesquels il/elle est plus à l'aise et/ou davantage convaincu, d'autres étais l'inspirant moins. Notre production est à voir comme un étai possible pouvant guider un travail de réflexion autour de la conception de dispositifs pédagogiques, incitant par exemple à se poser des questions essentielles (quelles dimensions est-il pertinent de travailler prioritairement comptetenu des caractéristiques du public en formation? Quelles articulations de ces dimensions si l'accompagnement s'inscrit dans la durée? Avec quelles dimensions aurais-je quelque chose d'intéressant à proposer, la possibilité de mobiliser des étais qui m'inspirent? etc.), et proposant des idées d'étais remobilisables, à adapter et à s'approprier.

En dépit de l'originalité de notre grille en termes de finalité, la dimension c que nous proposons et, dans une moindre mesure, la dimension e, pouvaient paraître relativement attendues au regard des définitions de la réflexivité que l'on trouve dans la littérature. Dans la dimension c, on entrevoit en effet la réflexivité comme une pratique très proche, par exemple, de ce qu'énonce Varga (2014) lorsqu'elle décrit la réflexivité comme une démarche cognitive imbriquant, dans un cheminement spiralaire, les trois éléments suivants : «déstructuration et mise à plat de l'expérience; compréhension et interprétation des situations et des acteurs; articulation des liens, production de significations personnelles (re)construction du sens ».

La présence de la dimension d est, quant à elle, bien en phase avec l'idée de Jorro (2005) selon laquelle la réflexivité est trop souvent limitée à sa dimension d'analyse et pas assez à sa dimension projective. Plus récemment, dans nos domaines, Chizallet, Barcellini et Prost (2018) ont également mis en évidence l'intérêt d'explorer les relations entre activités réflexives et activités projectives. Bien que, pour notre part, nous considérions les activités projectives comme étant partie intégrante de l'exercice réflexif (elles font l'objet de notre dimension d), cette observation de Chizallet et al. (2018) conforte bien l'enjeu de penser les aspects d'analyse et les aspects de projection de manière conjointe.

Finalement, des dimensions de la réflexivité proposées dans le modèle, les plus originales nous paraissent être les a et b. Leur présence $n^{\prime} e s t$ sans doute pas étrangère aux spécificités des milieux au sein desquels nous enseignons. Dans ces milieux, le fait de se considérer comme un sujet influencé en situation professionnelle par des perceptions, des valeurs, ou tout autre élément «non scientifique», ne va pas toujours de soi, ce qui rend, nous l'avons vu, la dimension b particulièrement pertinente à travailler. Par ailleurs, les résistances face à l'exercice réflexif peuvent être nombreuses : le mot « réflexivité » et l'explicitation ex ante du dispositif parfois en le référant aux champs de la psychologie ou/et de la sociologie, non seulement peuvent indisposer les apprenants, mais aussi parasiter, selon les étais mobilisés et la dimension abordée, la démarche. Dans de telles conditions, nous proposons, outre de ne pas mentionner (trop vite) le mot «réflexivité», de miser sur la curiosité des apprenants vis-à-vis de leurs pairs (dimension a) pour stimuler leur envie de s'engager dans les exercices.

\section{Un apport sur la question des résistances face à l'exercice réflexif?}

La grille que nous proposons, pensée en priorité pour les milieux de l'agronomie au sens large, au sein desquels les résistances face à l'exercice réflexif peuvent s'avérer nombreuses, ne pourrait-elle pas également nourrir la réflexion des concepteurs de dispositifs dans d'autres types de milieux professionnels? Plusieurs auteurs évoquent en effet la difficulté, pour certaines personnes, à entrer dans un exercice réflexif, y compris dans le «secteur des métiers de l'humain », pour reprendre nos propos introductifs. Jorro (2005) parle par exemple d'une «posture de retranchement»; Varga (2014) évoque des types de rapports aux autres et des types de rapports aux dispositifs de formation différents entre les individus, qui conditionnent la manière avec laquelle ils s'engagent dans les exercices réflexifs qu'on leur propose. Dans tous les cas, I'adoption ou non d'une authentique posture de réflexivité, définie par Morisse $(2014$, p. 24) comme «une posture de curiosité et de questionnement, portée par une volonté délibérative et critique», semble poser question, y compris dans des milieux au sein desquels le travail réflexif parait davantage, sinon accepté, du moins présent dans la culture commune. 
Le passage par les dimensions a et b de notre modèle, qui peuvent paraître plus triviales que les dimensions c, $d$ et e, mais y sont indubitablement liées, ne pourrait-il pas aider à lever certaines réticences face à l'exercice réflexif, en faisant naître, par une confrontation explicite à l'altérité, un intérêt susceptible de nourrir la posture de curiosité et de questionnement dont parle Morisse (2014)?

\section{En guise d'ouverture}

Le travail réflexif issu de cette prise de recul sur nos pratiques d'accompagnement d'apprenants, nous a amenés, en tant que formateurs, à réexplorer, pour nous-mêmes, chacune des cinq dimensions, et, ce faisant, à réexaminer la justesse de nos interventions dans ces accompagnements. Des changements de pratiques ont d'ores et déjà été engagés consécutivement à ce travail, qui pourraient faire l'objet de réflexions et de travaux futurs.

Rappelons enfin que notre grille, vue comme un cadre d'analyse dont nous nous dotons pour travailler sur nos dispositifs, est culturellement située, à de nombreux égards. En premier lieu, et comme déjà évoqué, parce que nous appartenons à un milieu professionnel donné. Mais la réflexion peut sans doute être poussée plus loin. L'expression même de nos dimensions de la réflexivité, et en particulier la dimension b («j'ai une certaine conscience d'être un sujet à part entière... »), est loin d'être neutre d'un point de vue philosophique. Pastré (2011) le rappelle bien dans son ouvrage sur la didactique professionnelle, en faisant référence à Heidegger :

C'est parce que les hommes sont des êtres qui sont capables de s'interroger sur leur mode d'être, c'est parce qu'ils ne sont pas seulement ce qu'ils sont (des «étants», dit Heidegger), qu'ils sont, en conséquence, des sujets. Et c'est parce qu'ils ont ce pouvoir d'interrogation sur eux-mêmes qu'ils sont aussi capables de renverser ce qui les affecte en traits identitaires assumés. (p. 132)

Cette posture philosophique, à laquelle nous souscrivons, est pourtant loin d'être universellement partagée. Spinoza par exemple considère qu'on ne peut pas construire une philosophie à partir du sujet, parce que ce dernier manque de consistance, idée reprise par Lévi-Strauss (1958, cité par Pastré, 2011, p. 129) : «le sujet, cet insupportable enfant gâté qui a trop longtemps occupé la scène philosophique, et empêché tout travail sérieux en réclamant une attention exclusive» (Pastré, 2011). Quelle place reste-t-il pour le sujet et pour la réflexivité si on s'adonne à une critique de l'anthropocentrisme? La question reste ouverte.

\section{Remerciements}

Merci à l'ensemble des étudiants-ingénieurs, doctorants et stagiaires, ainsi qu'aux coanimateurs de nos différentes sessions de formation, qui continuent chaque année à faire évoluer nos dispositifs et à enrichir de façon très vivante nos expériences pédagogiques d'accompagnement au développement professionnel par la réflexivité.

\section{Bibliographie}

Beaujouan, J., Coutarel, F., \& Daniellou, F. (2013). L'expérience des autres dans la formation. Apports et limites du récit professionnel. Éducation permanente, 196(3), 25-38.

Boal, A., Mellac, R., \& Rigot-Müller, V. (2004). Jeux pour acteurs et non-acteurs : pratique du théâtre de l'opprimé. Paris, France : La Découverte.

Boltanski, L., \& Thévenot, L. (1991). De la justification. Les économies de la grandeur. Paris, France : Gallimard.

Bourgeois, E. (2014). Les figures de l'apprentissage au travail au-delà de la réflexivité. Dans É. Bourgeois, \& S. Enlart (Eds.), Apprendre dans l'entreprise. (pp.147-165). Paris, France : Presses universitaires de France.

Bruner, J. (1998). Le développement de l'enfant. Savoir faire, savoir dire. Paris, France: Presses universitaires de France.

Buysse, A., \& Vanhulle, S. (2009). Écriture réflexive et développement professionnel : quels indicateurs? Questions vives, 5(11), 225-242. doi:10.4000/questionsvives.603.

Caron, J., \& Chouinard, I. (2015). Le recours aux approches réflexives dans les métiers relationnels : modélisation des conceptions de la réflexivité. Communication présentée au be Congrès de l'Association internationale pour la formation, la recherche et l'intervention sociale, Porto, Portugal.

Chizallet, M., Barcellini, F., \& Prost, L. (2018). Supporting farmers' management of change towards agroecological practices by focusing on their work: a contribution of ergonomics. Cahiers de l'Agriculture, 27 (3), 35005. doi :10.1051/cagri/2018023.

Clot, Y., \& Faïta, D. (2000). Genres et styles en analyse du travail : concepts et méthodes. Travailler, 4, 7-43.

Couturier, Y., (2013). Critique de la réflexivité (mais est-ce donc possible?). Phronesis, 2(1), 8-14. doi:10.7202/1015635ar. 
Donnadieu, B., (2001). Alternance : pour éviter la confusion des rôles et des postures. Dans P. Lhez, D. Millet, \& B. Séguier (Eds.), Alternance et complexité en formation. (pp. 133-146). Paris, France : Séli Arslan.

Fablet, D. (2004). Les groupes d'analyse des pratiques professionnelles : une visée avant tout formative. Connexions, 82(2), 105-117. doi:10.3917/cnx.082.0105.

Faingold, N., (2015). Un dispositif universitaire centré sur un travail réflexif d'exploration du vécu subjectif : formation et transformation de soi. Recherche et formation, 80, 47-62. doi:10.4000/rechercheformation.2499.

Gardet, J., (2016, 18 novembre). Le principe des cités de Boltanski et Thévenot. Repéré à https://www.youtube.com/ watch?v=Gy0G4nmWAAE.

Guillemette,F., (2016). La pratique réflexive:toutle monde en parle, mais... Approches inductives, 3(1), 1-6. doi:10.7202/1035192ar.

Hatton, N., \& Smith, D. (1995). Reflection in teacher education: towards definition and implementation. Teaching and teacher education, 11(1), 33-49. doi:10.1016/0742-051X(94)00012-U.

Hubert, B., (2004). Agricultures et développement durable, enjeux de connaissances et attitudes de recherche. Dossier de l'environnement de I'INRA, 27, 41-54.

Jorro, A. (2005). Réflexivité et auto-évaluation dans les pratiques enseignantes. Mesure et évaluation en éducation, 27(2), 33-47.

Lison, C. (2013). La pratique réflexive en enseignement supérieur : d'une approche théorique à une perspective de développement professionnel. Phronesis, 2(1), 15-27. doi:10.7202/1015636ar.

Morisse, M. (2014). Les dimensions réflexive et professionnalisante de l'écriture. Quelques considérations épistémologiques, théoriques et méthodologiques. Dans M. Morisse, \& L. Lafortune (Eds.), L'écriture réflexive, objet de recherche et de professionnalisation. (pp. 9-30). Sainte-Foy, Canada: Presses de l'Université du Québec.

Pastré, P. (2011). La didactique professionnelle. Approche anthropologique du développement chez les adultes. Paris, France: Presses universitaires de France.

Paul, M. (2016). La démarche d'accompagnement. Repères méthodologiques et ressources théoriques. Louvain-la-Neuve, Belgique : De Boeck Supérieur.

Rogers, C. (1968). Le développement de la personne. Paris, France : Dunod.

Sparks-Langer, G.M., Simmons, J.M., Pasch, M., Colton, A., \& Starko, A. (1990). Reflective pedagogical thinking: how can we promote it and measure it? Journal of teacher education, 41(5), 23-32.

Tardif, M., Borges, C., \& Malo, A. (2012). Le virage réflexif en éducation : où en sommes-nous 30 ans après Schön? Bruxelles, Belgique : De Boeck Supérieur.

Trognon, L. (2013). Accompagner la réflexivité, une construction de l'expérience. Éducation permanente, 196(3), 65-74.

Trognon, L. (2019). Accompagner la réflexivité des managers du développement territorial. Psychanalyse \& Management, 8 , 49-63.

Uwamariya, A., \& Mukamurera, J. (2005). Le concept de « développement professionnel » en enseignement : approches théoriques. Revue des sciences de l'éducation, 31(1), 133-155. doi:10.7202/012361ar.

Van Manen, M. (1977). Linking ways of knowing with ways of being practical. Curriculum Inquiry, 6, 205-228. doi:10.1080/03626 784.1977.11075533.

Varga, R. (2014). Manifestations linguistiques de la réflexivité et de la non-réflexivité : les postures en jeu dans la transformation du sujet. ¿ Interrogations?, 18, [en ligne], https://www.revue-interrogations.org/Manifestations-linguistiques-de-la (Consulté le 10 mai 2019).

Vermersch, P. (1994/2019). L'entretien d'explicitation. Paris, France : ESF.

Vial, M. (2006). Accompagner n'est pas guider. Communication présentée à la Conférence aux formateurs de l'Ecole de la Léchère, Fondation pour les classes d'enseignement spécialisé de la Gruyère, Suisse. 